\title{
Análisis espacio temporal (1981-2010) de la precipitación en la ciudad de Bogotá: avances en la generación de índices extremos
}

\author{
Juan Antonio Aragón-Moreno ${ }^{1}$ \\ Brayan David Lerma-Lerma ${ }^{2}$
}

Fecha de recepción: 3 de enero de 2019

Fecha de aprobación: 21 de marzo de 2019

\begin{abstract}
Resumen
La investigación analizó los escenarios espaciales y temporales de eventos extremos de precipitación en Bogotá, Colombia, a partir del desarrollo de los índices de extremos climáticos avalados por el Panel Intergubernamental del Cambio Climático (IPCC) y por su capacidad de predicción de tendencias climáticas. Se trabajaron datos de frecuencia diaria y registros de treinta años de 23 estaciones meteorológicas distribuidas ampliamente en la ciudad, con el fin de brindar la mayor cantidad de información espacial. Se controló la calidad y ausencia de datos. El perfil climatológico reveló dos regímenes pluviométricos mensuales en el área de estudio con valores de hasta $87.2 \mathrm{~mm} /$ día de precipitación, además se encontraron dos amplias zonas de convergencia de precipitación. Los índices evidenciaron una tendencia positiva regional salvo en el sur de la ciudad que se presentaron tendencias negativas, lo que proyectó notables cambios en la intensidad, duración y frecuencia de eventos extremos, de igual manera el análisis geoestadístico detalló la zona oriental con un amplio matiz en la dinámica hidrológica, apropiada para explorarse en futuras investigaciones. En última instancia, esta investigación se orienta en ampliar y divulgar información clave en la planeación y ordenación del territorio, así como también en la prevención y gestión del riesgo frente a escenarios de extremos climáticos como deslizamientos de tierra, inundaciones o sequias en ciudades situadas por encima de los 2000 m.s.n.m. y de esta manera ayudar a tomadores de decisiones, investigadores, profesores y estudiantes universitarios en emplear una metodología aprobada internacionalmente que unifica criterios para el cotejo de información climática.
\end{abstract}

Palabras clave: análisis estadístico; cambio climático; climatología; lluvia; pronóstico; toma de decisiones.

\section{Analysis temporary space (1981-2010) of the precipitation in the city of Bogota: advances in the generation of extreme indices}

\begin{abstract}
The investigation analyzed the spatial and temporal scenarios of extreme precipitation events in Bogotá, Colombia, based on the development of climate end indexes endorsed by the Intergovernmental Panel on Climate Change (IPCC) and their capacity to predict climate trends. Data of daily frequency and records of thirty years of 23 meteorological stations distributed widely in the city were worked, in order to provide the greatest amount of spatial information. The quality and absence of data were controlled. The climatological profile revealed two monthly rainfall regimes in the study area with values of up to $87.2 \mathrm{~mm} /$ day of precipitation, in addition two broad areas of precipitation convergence were found. The indexes showed a positive regional trend except in the south of the city where negative trends were presented, which projected
\end{abstract}

\footnotetext{
${ }^{1}$ M. Sc. Universidad Libre (Bogotá-Distrito Capital, Colombia). juan.aragon@unilibrebog.edu.co. ORCID: 0000-00032768-9082.

$\frac{27}{2}$ Universidad Libre (Bogotá-Distrito Capital, Colombia). brayand-lermal@unilibre.edu.co. ORCID: 0000-0001-93648030.

Revista Facultad de Ingeniería (Rev. Fac. Ing.) Vol. 28 (51), pp. 51-71. Abril-Junio 2019. Tunja-Boyacá, Colombia. L-ISSN: 0121-1129, e-ISSN: 2357-5328, DOI: https://doi.org/10.19053/01211129.v28.n51.2019.9123
} 
notable changes in the intensity, duration and frequency of extreme events, in the same way the geostatistical analysis detailed the eastern area with a wide nuance in hydrological dynamics, appropriate to be explored in future research. Ultimately, this research is aimed at expanding and disseminating key information in the planning and planning of the territory, as well as in the prevention and management of risk in the face of extreme weather scenarios such as landslides, floods or droughts in cities located by above 2000 m.a.s.l. and in this way help decision makers, researchers, professors and university students to use an internationally approved methodology that unifies criteria for the collation of climate information.

Keywords: climate change; climatology; decision making; forecasting; rainfall; statistical analysis.

\section{Análise espaço temporal (1981-2010) da precipitação na cidade de Bogotá: avanços na geração de índices extremos}

\section{Resumo}

A pesquisa analisou os cenários espaciais e temporais de eventos extremos de precipitação em Bogotá, Colômbia, a partir do desenvolvimento dos índices de extremos climáticos avalizados pelo Painel Intergovernamental da Mudança Climática (IPCC) e por sua capacidade de predição de tendências climáticas. Trabalharam-se dados de frequência diária e registros de trinta anos de 23 estações meteorológicas distribuídas amplamente na cidade, com o fim de brindar a maior quantidade de informação espacial. Controlou-se a qualidade e a ausência de dados. O perfil climatológico revelou dois regimes pluviométricos mensais na área de estudo com valores de até $87.2 \mathrm{~mm} /$ dia de precipitação, além disso, encontraram-se duas amplas zonas de convergência de precipitação. Os índices evidenciaram uma tendência positiva regional salvo no sul da cidade que se apresentaram tendências negativas, 0 que projetou notáveis mudanças na intensidade, duração e frequência de eventos extremos, de igual maneira a análise geoestatística detalhou a zona oriental com um amplo matiz na dinâmica hidrológica, apropriada para explorar-se em futuras pesquisas. Em última instância, esta pesquisa se orienta em ampliar e divulgar informação fundamental no planejamento e ordenação do território, assim como também na prevenção e gestão de risco frente a cenários de extremos climáticos como desmoronamentos de terra, inundações ou estiagens em cidades situadas acima dos 2000 m.s.n.m. e desta maneira ajudar aos que tomam decisões, pesquisadores, professores e estudantes universitários a empregar uma metodologia aprovada internacionalmente que unifica critérios para a comparação de informação climática.

Palavras chave: análise estatística; mudança climática; climatologia; chuva; prognóstico; tomada de decisões.

\section{Para citar este artículo:}

J. A. Aragón-Moreno, and B. D. Lerma-Lerma, "Análisis espacio temporal (1981-2010) de la precipitación en la ciudad de Bogotá: avances en la generación de índices extremos," Revista Facultad de Ingeniería, vol. 28 (51), pp. 51-71, Abr. 2019. DOI: https://doi.org/10.19053/01211129.v28.n51.2019.9123.

Esta obra está bajo licencia internacional Creative Commons Reconocimiento 4.0

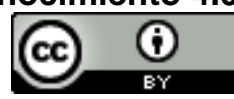




\section{INTRODUCCIÓN}

De acuerdo con la tendencia de precipitación extrema en Suramérica, en Colombia la frecuencia de eventos extremos no tiene una tendencia definida [13]. Siendo vital conocer a detalle la dinámica espacial y temporal de la precipitación, debido a que es la principal entrada de agua a una cuenca, por lo que su comportamiento repercute directa o indirectamente en las actividades económicas de ciudades de gran altura como Tunja, Pasto o la sabana de Bogotá [4-5] hasta incluso ciudades cercanas al nivel del mar [6] .

En la ciudad de Bogotá, han ocurrido eventos climáticos extremos de gran magnitud, por ejemplo en marzo de 1988 se presentaron inundaciones en diversos barrios de la ciudad por fuertes lluvias, mientras que tan solo cuatro años después en 1992 una fuerte sequía generó problemas de racionamiento energético, dado que la falta de lluvias afectó el nivel de los embalses de las hidroeléctricas [7]. De manera más reciente, la granizada del 3 de noviembre de 2007 cuya precipitación fue de $98 \mathrm{~mm}$ ese día [8], dejo daños económicos estimados de 16.000 millones de pesos, cortes eléctricos y evacuaciones de múltiples hogares [4].

Los índices extremos son ciertos cálculos numéricos, que se hacen a partir de una serie de datos de un parámetro y que permiten conocer la tendencia del parámetro. Sabiendo de esta manera si la variable ha aumentado o disminuido en un lugar específico [5]. Convirtiéndose en una herramienta potencial para la predicción de brotes infecciosos de enfermedades en zonas tropicales [11], pronósticos en el clima para zonas de agricultura [12] así como también aportar información científica en el ordenamiento de cuencas y territorios por medio de perfiles climáticos del área de estudio [13].

En este orden de ideas, se han realizado estudios enfocados en evaluar el comportamiento de la precipitación por medio de índices de extremos climáticos para Colombia por el Instituto de Hidrología, Meteorología y Estudios Ambientales (IDEAM) [14] y el realizado por la Alcaldía de Bogotá en conjunto con otras instituciones por medio de la metodología STAtistical and Regional Dynamical Downscaling of EXtremes -STARDEX-para la región de Bogotá y Cundinamarca, el cual establece la precipitación promedio de la región de Bogotá tuvo un aumento de $0.3 \mathrm{~mm}$ por década sin embargo, las precipitaciones extremas tuvieron un aumento de entre 1 a $2 \mathrm{~mm}$ por década, esto va de la mano con el hecho de que la máxima precipitación en 5 días seguidos incremente de 6 a $9 \mathrm{~mm}$ por década, esperando lluvias más extremas en esta región [10].

El estudio realizado por Losada [16] es el único desarrollado para Bogotá, que establece que la tendencia de días con precipitaciones mayores a $20 \mathrm{~mm}$ aumentarán en un rango entre 0.061 y 0.391 día/año [17].

Por otra parte, dentro de los métodos de interpolación espacial para la variable precipitación se encuentran el estudio desarrollado por Vargas, Santos, Cárdenas, \& Obregón [18] el cual enfatiza en que el método de inverso de la distancia ponderada es el que mejor se adapta a las condiciones de la ciudad, 
sin embargo otros investigadores reportan para otras partes del mundo resultados favorables como la interpolación Kriging [19], incluso para otros métodos como el Spline y Vecino natural [15].

En síntesis, este trabajo tiene como objetivo analizar el comportamiento espacial y temporal de la precipitación, en una ciudad situada por encima de los 2000 m.s.n.m. en conjunto con índices extremos de precipitación para generar información base en la toma de decisiones de planeación territorial, gestión y prevención del riesgo.

\section{MATERIALES Y MÉTODOS}

\section{A. Área de estudio y recolección datos}

Bogotá está localizada en la cordillera oriental de Colombia, con una extensión cercana de $33 \mathrm{~km}$ de norte a sur y de $16 \mathrm{~km}$ de occidente a oriente y una altura media de 2630 m.s.n.m.; sus coordenadas comprenden 4³5'56" Latitud Norte y $74^{\circ} 04^{\prime} 51^{\prime \prime}$ Longitud Oeste según el observatorio astronómico de Bogotá [21].

Se obtuvieron 23 estaciones meteorológicas (Figura 1) con información de precipitación total diaria de tres entidades pertenecientes al (IDEAM), la Empresa de Acueducto y Alcantarillado de Bogotá (EAAB) y la Corporación Autónoma Regional de Cundinamarca (CAR).

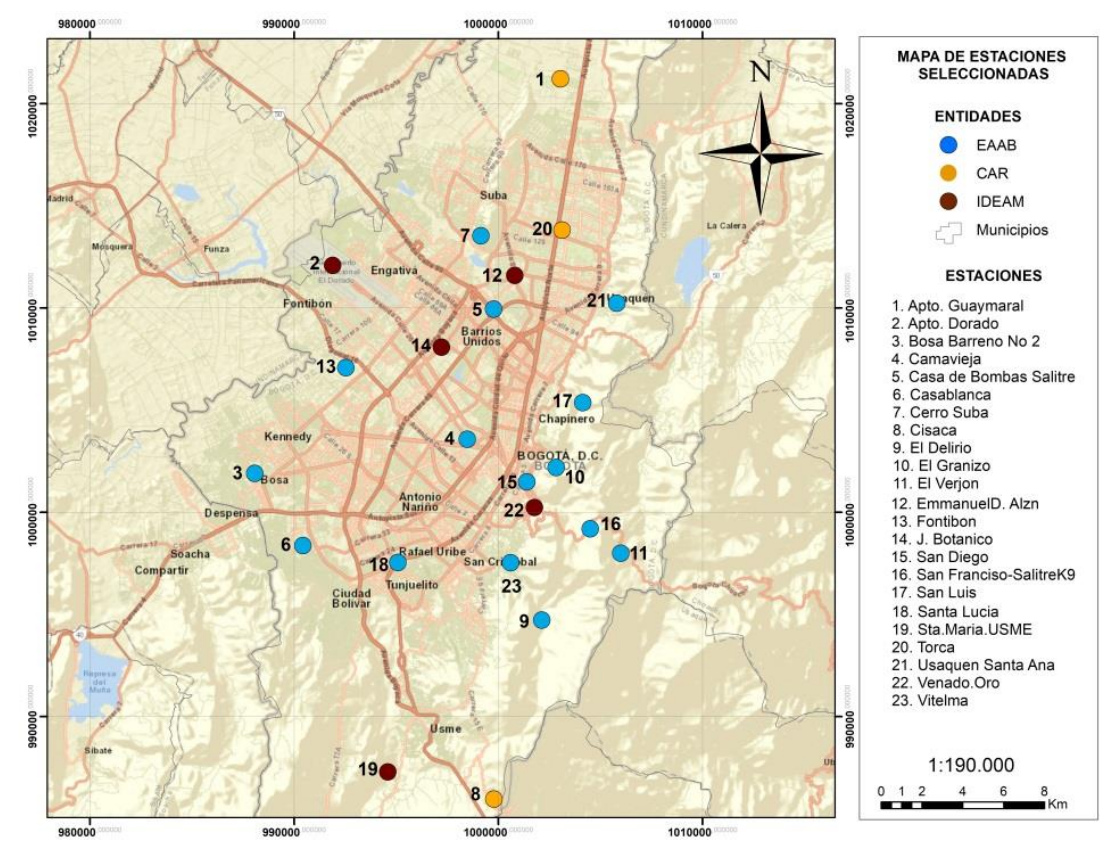

Fig. 1. Ubicación geográfica de las estaciones meteorológicas seleccionadas.

Adicionalmente se utilizaron cinco estaciones meteorológicas de temperatura diaria máxima y mínima las cuales fueron Jardín Botánico, Aeropuerto el Dorado, Venado de oro, San Jorge y Aeropuerto Guaymaral, empleadas para el llenado de datos faltantes [22].

La ciudad de Bogotá posee un régimen de lluvia bimodal con dos periodos húmedos; el primero representado por los meses de marzo, abril, mayo (MAM) 
y el segundo correspondiente a los meses de septiembre, octubre y noviembre (SON). Por otra parte, cuenta con dos periodos secos; el primero corresponde a los meses de diciembre, enero, febrero (DEF) y el segundo representado por los meses de junio, julio, agosto (JJA), a pesar de ello existe un régimen monomodal poco acentuado en la parte rural posterior a los cerros orientales, atribuido a que la orografía del terreno se ve influenciada por el régimen de lluvias de los llanos orientales [23-25]. Sin embargo, existe una sectorización de la precipitación en la ciudad, de acuerdo con la normal climatológica de 1971 - 2000 donde la precipitación anual promedio de la ciudad, en la zona oriental los valores oscilan entre 900 a $1100 \mathrm{~mm}$, influenciados principalmente por los cerros orientales, por otra parte el norte de la ciudad posee valores de 700 a $900 \mathrm{~mm}$ y en la zona occidental las precipitaciones son entre 600 y 700 $\mathrm{mm}$ con variaciones entre 700 a $800 \mathrm{~mm}$ en el noroccidente [24].

\section{B. Control de calidad de datos de precipitación}

Los datos de estaciones meteorológicas, normalmente contienen algunos errores, debido a que los datos algunas veces no tienen un buen manejo o también faltan periodos de tiempo por lo que hay vacíos en las series de tiempo [26-27].En relación con esto, hay técnicas de control de calidad de datos, una de ellas se establecer un criterio de validación de datos, el cual consistió en utilizar tres desviaciones estándar y un rango de datos que estuvieran por encima del primer cuartil -Q1- y por debajo del tercer cuartil $-Q_{2}$ - para ello se usó Software RClimtool [28] que cuenta con capacidad de realizar el análisis.

Adicionalmente se comparó la información de precipitación media total mensual de Bogotá, proveniente del atlas climatológico de Colombia para la normal de 1981-2010 [29]. De esta manera para cada mes, se detectaron y depuraron los valores diarios que excedieran la precipitación media mensual o que de alguna manera fueran atípicos para ese periodo del año.

\section{Llenado de datos faltantes}

Se siguieron las recomendaciones de la Organización Meteorológica Mundial para la estimación de datos faltantes de acuerdo a la Guía de prácticas climatológicas [30] los métodos empleados fueron: correlación de Pearson donde el valor de la regresión lineal debía ser mayor de 0.7 el adecuado para completar series [31-32]. El segundo método correspondiente al llenado por curvas de dobles masas que permite llenar datos a través de un proceso estadístico y gráfico [33].

El último método, fue el de estimaciones por medio de modelos VAR el cual consiste en usar información de las estaciones meteorológicas de temperatura máxima y mínima para llenar datos de precipitación [28]. Sin embargo, dado que el número de estaciones de precipitación era mayor que las de temperatura máxima y mínima, se recurrió a utilizar polígonos de Thieseen [34] para agrupar las estaciones, de esta manera la información de temperatura máxima y mínima de una misma estación se empleó para llenar varias estaciones de precipitación, de acuerdo a la representatividad espacial de cada estación. 
Análisis espacio temporal (1981-2010) de la precipitación en la ciudad de Bogotá: avances en la generación de índices extremos

\section{Análisis estadístico}

Se realizó el análisis descriptivo univariado [35] para la exploración de datos de precipitación diaria multianual para cada estación por medio de cuartiles, medidas de tendencia central, varianza, rangos, máximos y mínimos, posteriormente se aplicó el análisis para valores mensuales multianuales de cada una de las estaciones y así poder clasificar por grupos las estaciones meteorológicas de acuerdo al comportamiento mensual multianual del régimen pluviométrico y a sus propiedades estadísticas. Adicionalmente se requirió la herramienta de diagramas Boxplot [36] para sintetizar la gran cantidad de datos de forma visual. Las gráficas se generaron por medio del software Statgrapichs Centurion $16 \AA$.

\section{E. Índices para eventos extremos}

Para conseguir una perspectiva uniforme sobre los cambios observados en los extremos climáticos, el Equipo en Detección de Cambio Climático e Índice determinó un paquete básico de índices de extremos, el grupo básico incluye 27 índices extremos de temperatura y precipitación [37], los cuales trabajan en el software RClimdex desarrollados por el ETCCDI [17]. Para el caso de esta investigación se trabajó con 10 índices del grupo básico (Tabla I) correspondientes a los de precipitación.

Tabla I. Índices de precipitación extrema. Adaptado de [37],

\begin{tabular}{|c|c|c|c|}
\hline ID & Nombre & Definición & Unidad \\
\hline RX1day & $\begin{array}{l}\text { Cantidad máxima } \\
\text { precipitación en un día }\end{array}$ & $\begin{array}{l}\text { Máximo mensual } \\
\text { precipitación en } 1 \text { día }\end{array}$ & $\mathrm{mm}$ \\
\hline RX5day & $\begin{array}{l}\text { Cantidad máxima de } \\
\text { precipitación en } 5 \text { días }\end{array}$ & $\begin{array}{lccc}\text { Máximo } & \text { mensual } & \text { de } \\
\text { precipitación } & \text { en } 5 & \text { días } \\
\text { consecutivos } & & & \end{array}$ & $\mathrm{mm}$ \\
\hline R5 & Número de días sobre $5 \mathrm{~mm}$ & $\begin{array}{l}\text { Número de días en un año en } \\
\text { que } P R C P>=5 \mathrm{~mm}\end{array}$ & días \\
\hline $\mathrm{R} 10$ & $\begin{array}{l}\text { Número de días con } \\
\text { precipitación intensa }\end{array}$ & $\begin{array}{l}\text { Número de días en un año en } \\
\text { que prcp }>=10 \mathrm{~mm}\end{array}$ & días \\
\hline R20 & $\begin{array}{l}\text { Número de días con } \\
\text { precipitación muy intensa }\end{array}$ & $\begin{array}{l}\text { Número de días en un año en } \\
\text { que prcp }>=20 \mathrm{~mm}\end{array}$ & días \\
\hline CDD & Días secos consecutivos & $\begin{array}{l}\text { Número máximo de días } \\
\text { consecutivos con prcp }<1 \mathrm{~mm}\end{array}$ & días \\
\hline CWD & Días húmedos consecutivos & $\begin{array}{l}\text { Número máximo de días } \\
\text { consecutivos con prcp }>=1 \mathrm{~mm}\end{array}$ & días \\
\hline R95p & Días muy húmedos & $\begin{array}{l}\text { Precipitación anual total en } \\
\text { que prcp>95 percentil }\end{array}$ & $\mathrm{mm}$ \\
\hline R99p & extremadamente & $\begin{array}{l}\text { Precipitación anual total en } \\
\text { que prcp > } 99 \text { percentil }\end{array}$ & $\mathrm{mm}$ \\
\hline PRCPTOT & $\begin{array}{l}\text { Precipitación total anual en } \\
\text { los días húmedos }\end{array}$ & $\begin{array}{l}\text { Precipitación anual total en los } \\
\text { días húmedos prcp }>=1 \mathrm{~mm}\end{array}$ & $\mathrm{~mm}$ \\
\hline
\end{tabular}

\section{F. Espacialización de información}

Se trabajaron cuatro interpolaciones diferentes, Kriging lineal, Vecino natural, Spline [15] e Inverso de la distancia ponderada (IDW) que presenta mejores características para Bogotá [18]. A su vez, se escogió la interpolación más adecuada con los datos promedios de precipitación anual de cada una de las 
estaciones para posteriormente emplear dicha interpolación con los datos de precipitación media trimestral y las tendencias para cada índice extremo. Los mapas se generaron con ArcGis 10.3.

\section{Resultados y Discusión}

\section{A. Llenado de datos y análisis estadístico}

Con los modelos VAR, se llenaron por completo las series de tiempo, permitiendo rellenar un total de 24470 datos faltantes que contenían las 23 estaciones, en el periodo de estudio. En la Figura 2, se observa el método de llenado de la estación Fontibón, que tenía ausencia de 517 días en datos faltantes en toda la serie de tiempo, en rojo los datos proporcionados y en azul los llenados.

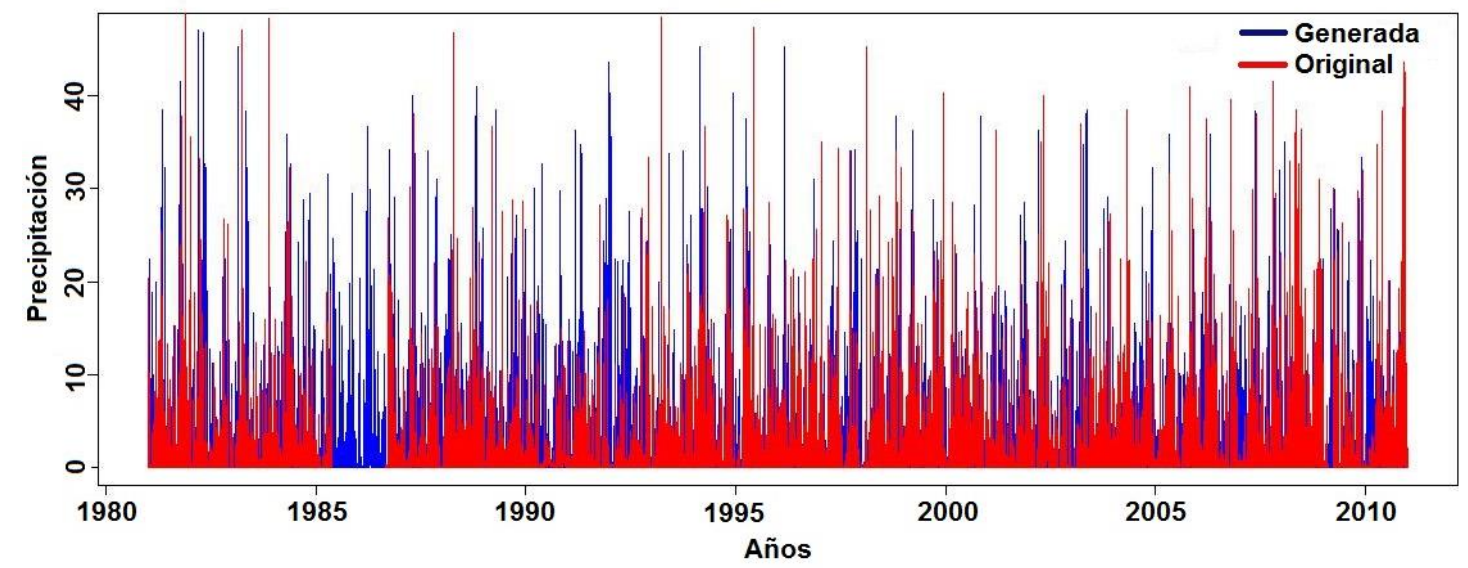

Fig. 2. Estación 13 con llenado de datos.

La descripción estadística reveló que los valores medios diarios de lluvia variaron de 1.7 a $3.5 \mathrm{~mm}$ (Tabla II). Adicionalmente la precipitación oscila de 1.3 a $4.4 \mathrm{~mm}$ en un $75 \%$ de los casos, presentado máximos diarios en la ciudad de 52.2 hasta $87.2 \mathrm{~mm}$ de precipitación. Obteniendo de esta manera una distribución que no es simétrica, donde la media es muy cercana al tercer cuartil y muy superior a la mediana (50\% de los datos), adicionalmente se señala que el comportamiento observado diario es análogo al del nivel mensual.

Tabla Il. Estadísticos de precipitación diaria de todas las estaciones en mm/día.

\begin{tabular}{|c|c|c|c|c|c|c|c|}
\hline Estación & Media & Desv. Estandar & $\mathbf{Q}_{1}$ & $\mathbf{Q}_{3}$ & Máximo & Mediana & Régimen \\
\hline 1 & 2.2 & 5.2 & 0 & 2.0 & 69.4 & 0 & Bimodal B \\
\hline 2 & 2.3 & 5.1 & 0 & 2.0 & 56.5 & 0.1 & Bimodal B \\
\hline 3 & 1.7 & 4.1 & 0 & 2.0 & 53.2 & 0 & Bimodal A \\
\hline 4 & 2.5 & 5.8 & 0 & 2.0 & 65.5 & 0.1 & Bimodal B \\
\hline 5 & 2.7 & 6.1 & 0 & 2.2 & 75.0 & 0.1 & Bimodal B \\
\hline 6 & 1.7 & 4.3 & 0 & 1.3 & 57.6 & 0.1 & Bimodal A \\
\hline 7 & 2.5 & 5.6 & 0 & 2.1 & 58.0 & 0.2 & Bimodal B \\
\hline 8 & 2.8 & 5.4 & 0 & 3.4 & 62.4 & 0.4 & Monomodal \\
\hline 9 & 3.5 & 6.2 & 0 & 4.2 & 64.0 & 0.7 & Monomodal \\
\hline 10 & 3.1 & 6.4 & 0 & 3.1 & 75.6 & 0.5 & Bimodal C \\
\hline 11 & 3.5 & 6.1 & 0 & 4.4 & 87.2 & 1.0 & Monomodal \\
\hline 12 & 2.4 & 5.9 & 0 & 1.6 & 62.4 & 0 & Bimodal B \\
\hline
\end{tabular}

Revista Facultad de Ingeniería (Rev. Fac. Ing.) Vol. 28 (51), pp. 51-71. Abril-Junio 2019. Tunja-Boyacá, Colombia. L-ISSN: 0121-1129, e-ISSN: 2357-5328, DOI: 
Análisis espacio temporal (1981-2010) de la precipitación en la ciudad de Bogotá: avances en la generación de índices extremos

\begin{tabular}{|c|c|c|c|c|c|c|c|}
\hline Estación & Media & Desv.Estandar & $\mathbf{Q}_{1}$ & $\mathbf{Q}_{3}$ & Máximo & Mediana & Régimen \\
\hline 13 & 2.1 & 4.7 & 0 & 1.8 & 53.6 & 0.2 & Bimodal B \\
\hline 14 & 3.2 & 6.7 & 0 & 3.0 & 67.8 & 0.3 & Bimodal C \\
\hline 15 & 3.0 & 6.6 & 0 & 2.4 & 80.6 & 0.3 & Bimodal C \\
\hline 16 & 3.4 & 6.4 & 0 & 3.9 & 66.9 & 0.7 & Monomodal \\
\hline 17 & 3.0 & 6.6 & 0 & 2.5 & 81.3 & 0.4 & Bimodal C \\
\hline 18 & 2.0 & 4.8 & 0 & 3.9 & 52.2 & 0.1 & Bimodal B \\
\hline 19 & 1.8 & 4.0 & 0 & 1.8 & 45.3 & 0.1 & Bimodal A \\
\hline 20 & 2.7 & 6.0 & 0 & 2.7 & 64.0 & 0 & Bimodal B \\
\hline 21 & 2.7 & 6.2 & 0 & 2.0 & 68.8 & 0.2 & Bimodal B \\
\hline 22 & 3.2 & 7.0 & 0 & 2.9 & 62.0 & 0.3 & Bimodal C \\
\hline 23 & 3.1 & 6.4 & 0 & 3.0 & 65.5 & 0.4 & Bimodal C \\
\hline
\end{tabular}

Por otra parte, el régimen pluviométrico mensual total de la ciudad, muestra dos comportamientos, uno monomodal poco acentuado para las estaciones 11(Figura 3),, 16, 9 y 8 alternándose entre abril , mayo y octubre los mayores registros de precipitación para cada estación, siendo $87.2 \mathrm{~mm} /$ día el máximo para el mes de mayo; y otro, bimodal para las 19 estaciones restantes, coincidiendo con lo reportado por Bernal et al [33], con la primera concentración de datos entre abril y mayo, y la segunda entre octubre y noviembre.

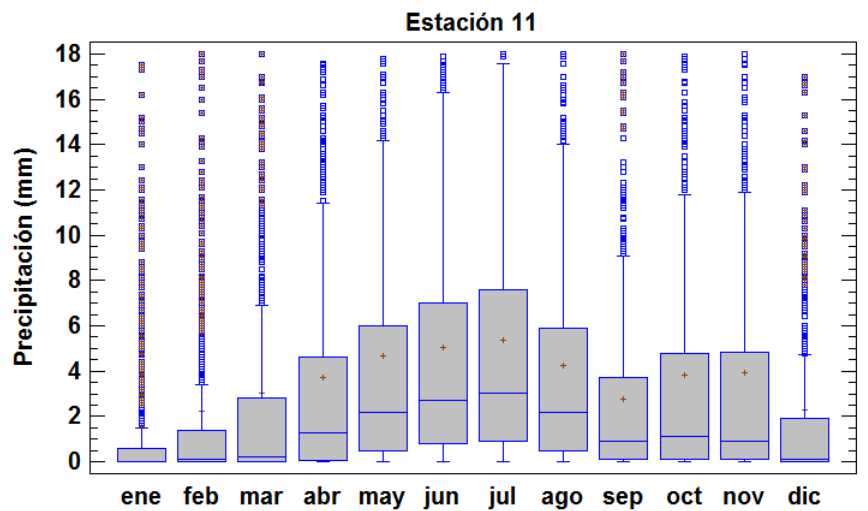

Fig. 3. Ejemplo Boxplot de estaciones con régimen monomodal.

Sin embargo, el grupo del régimen bimodal se subdividió en tres subgrupos de acuerdo con los valores medios diarios (Tabla II) que compartían rangos similares, presentándose así; bimodal $A$ donde los valores medios son menores a $2 \mathrm{~mm}$, bimodal B con valores medios entre 2 y $2.9 \mathrm{~mm}$ y bimodal $\mathrm{C}$ con valores medios mayores o iguales a $3 \mathrm{~mm}$. Las figuras 4, 5 y 6 presentan una estación de ejemplo por cada grupo. 


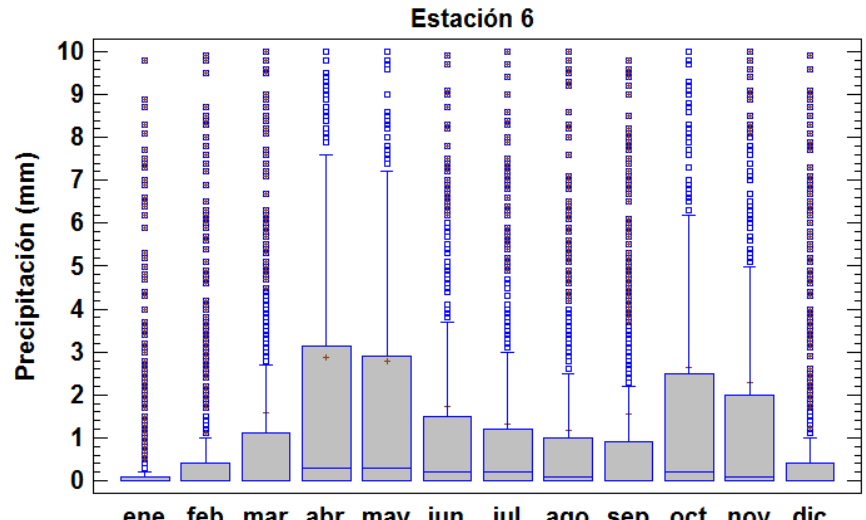

Fig. 4. Ejemplo Boxplot de grupo bimodal $A$.

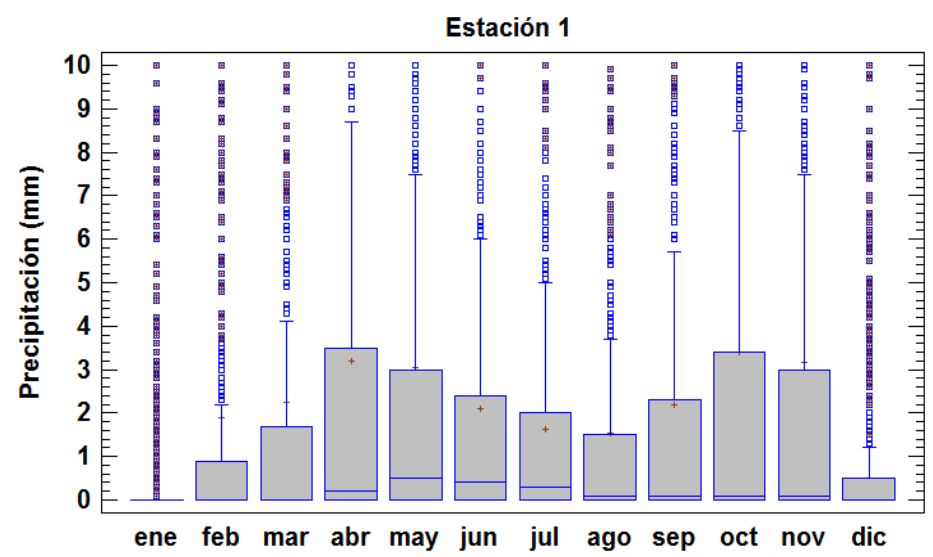

Fig. 5. Ejemplo Boxplot de grupo bimodal B.

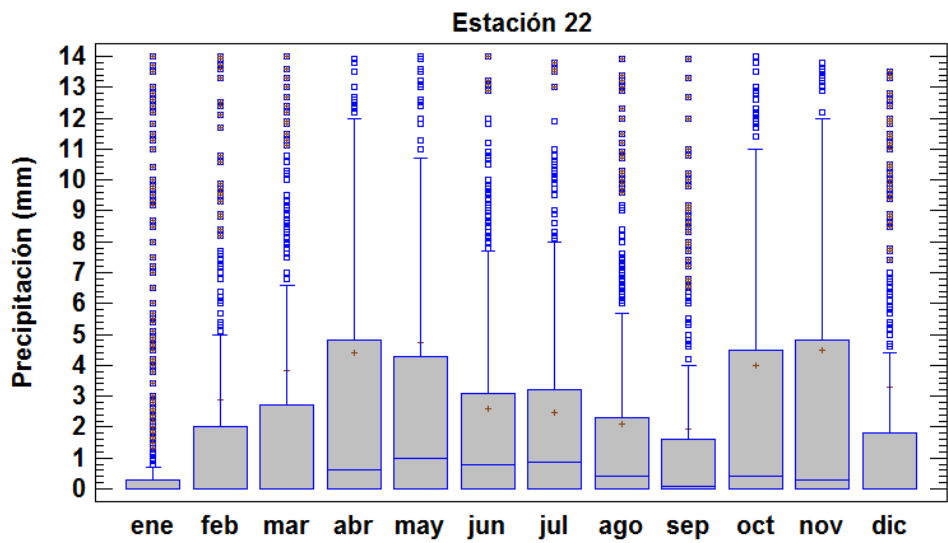

Fig. 6. Ejemplo Boxplot de grupo bimodal C.

Teniendo en cuenta lo anterior se definió que la máxima precipitación en bimodal A, B y C, alcanza valores de $57.6,75$ y $81.3 \mathrm{~mm} /$ día respectivamente, estas lluvias máximas corresponden a los meses de abril, mayo y noviembre los cuales son los meses con mayor número de días de lluvia para el grupo bimodal, sin embargo, para el grupo monomodal el periodo de junio y julio es el de mayor número de días con lluvias a pesar de que en estos meses no se presenten las máximas precipitaciones diarias. 
Análisis espacio temporal (1981-2010) de la precipitación en la ciudad de Bogotá: avances en la generación de índices extremos

\section{B. Índices para eventos extremos}

Se encontró que las 23 estaciones la variación fue positiva y negativa en la tendencia de los índices de manera general, con la excepción de la estación 8 que presento una tendencia negativa para la mayoría de los índices con la salvedad del índice CDD, el cual fue positivo.

A continuación, se presentan los índices extremos, los ejemplos gráficos estadísticos, corresponden a la estación de menor y mayor tendencia obtenida en cada índice donde la línea negra continua es la pendiente y la discontinua el comportamiento de la variable.

En cuanto a la precipitación máxima en un día (Rx1day) se encontraron valores de crecimiento entre 0.3 hasta $7 \mathrm{~mm} /$ década para 18 estaciones; por el contrario, las estaciones $3,6,8$ y 19, ubicadas en la periferia sur y occidental de la ciudad, reportan descensos entre -0.03 hasta $-1.7 \mathrm{~mm} /$ década, indicando un aumento en la zona concéntrica de la ciudad y una disminución de volumen de lluvia para el sur y occidente de la ciudad. Para la Figura 7 , la tendencia de la estación 10 es de $7 \mathrm{~mm} /$ década y $-1.7 \mathrm{~mm} /$ década para la estación 8 , sin embargo, en esta última estación hacia la última década los datos presentan una oscilación de crecimiento.

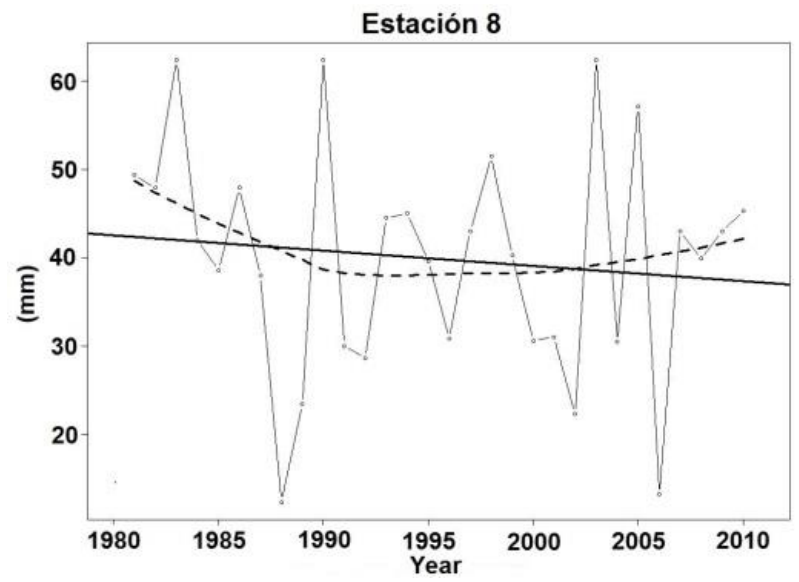

Fig. 7. Ejemplo de resultados índice Rx1day.

De manera similar el volumen de precipitación máxima en cinco días seguidos (Rx5day) aumentará entre 1 hasta $12 \mathrm{~mm} /$ década para 21 estaciones, siendo el valor máximo el de la estación 10 (Figura 8), coincidiendo con lo afirmado por IDEAM et al. [10], con excepción de las estaciones 8 (Figura 8 ) y 20, que reportan valores de -8 y -2 mm/década respectivamente, esperando un aumento de precipitaciones en la mayoría de la ciudad a excepción de la zona sur. 

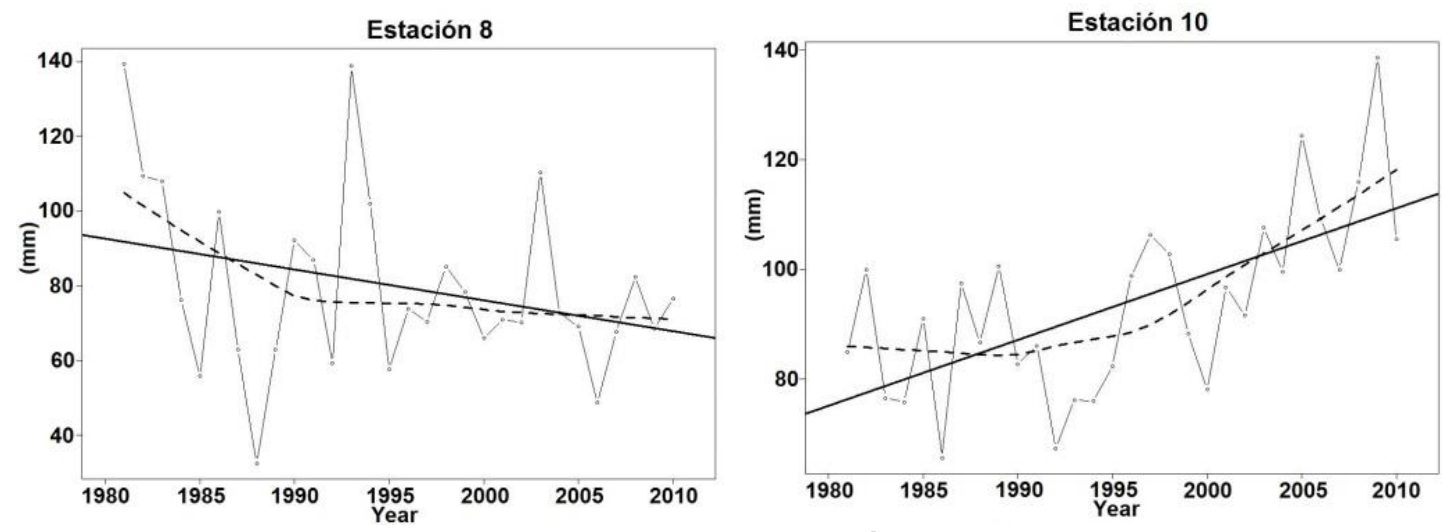

Fig. 8. Ejemplo de resultados índice Rx5day.

Para los índices R5, R10 y R20, la estación 8 presenta valores de -6, -3 y -1 días/década respectivamente proyectando que en una década habrá un día menos con precipitaciones mayores a $20 \mathrm{~mm}$, por el contrario las restantes 22 estaciones tienen tendencias ascendentes con picos máximos de 9,6 y 3 días/década respectivamente correspondiendo a las estaciones 14 y 17, lo que significa que en una década habrán tres días más con precipitaciones mayores a $20 \mathrm{~mm}$, coincidiendo con el rango reportado por Losada [16] para este valor. En las Figuras 9, 10 y 11 se presentan ejemplos de las estaciones por cada índice.
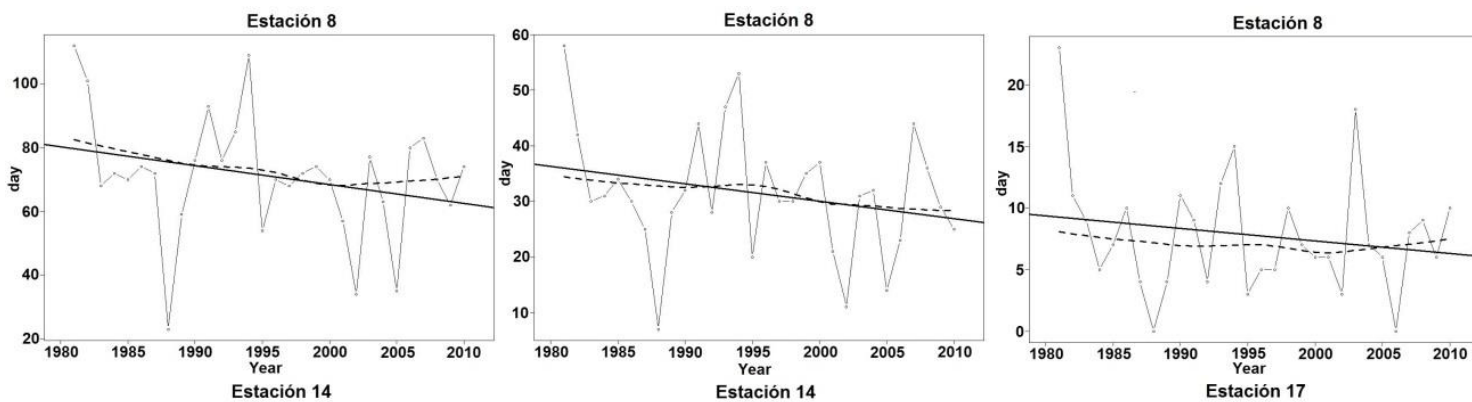

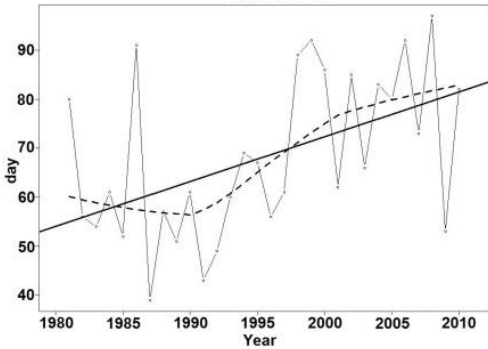

Fig. 9. Ejemplo de resultados índice $\mathrm{R} 5$.

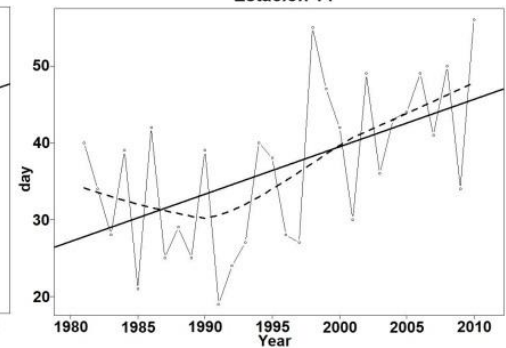

Fig. 10. Ejemplo de resultados índice $\mathrm{R} 10$.

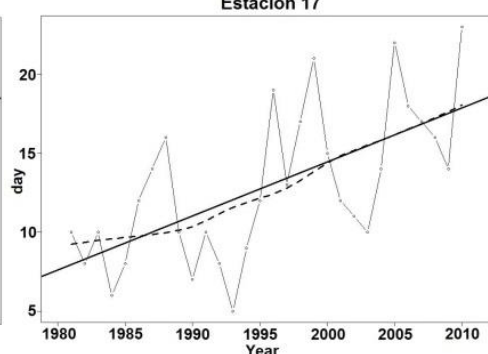

Fig. 11. Ejemplo de resultados índice R20.

El índice de días secos consecutivos presentó una tendencia positiva para las estaciones $4,8,9,10,11,16,17,19,20$ y 23 con crecimiento de hasta 8.1 días/década en la estación 19 (Figura 12) mientras que para las 13 estaciones restantes la tendencia negativa puede llegar a -3.6 días/década en la estación 7 (Figura 12) lo que significa menos días secos consecutivos para estas últimas estaciones. 
Análisis espacio temporal (1981-2010) de la precipitación en la ciudad de Bogotá: avances en la generación de índices extremos
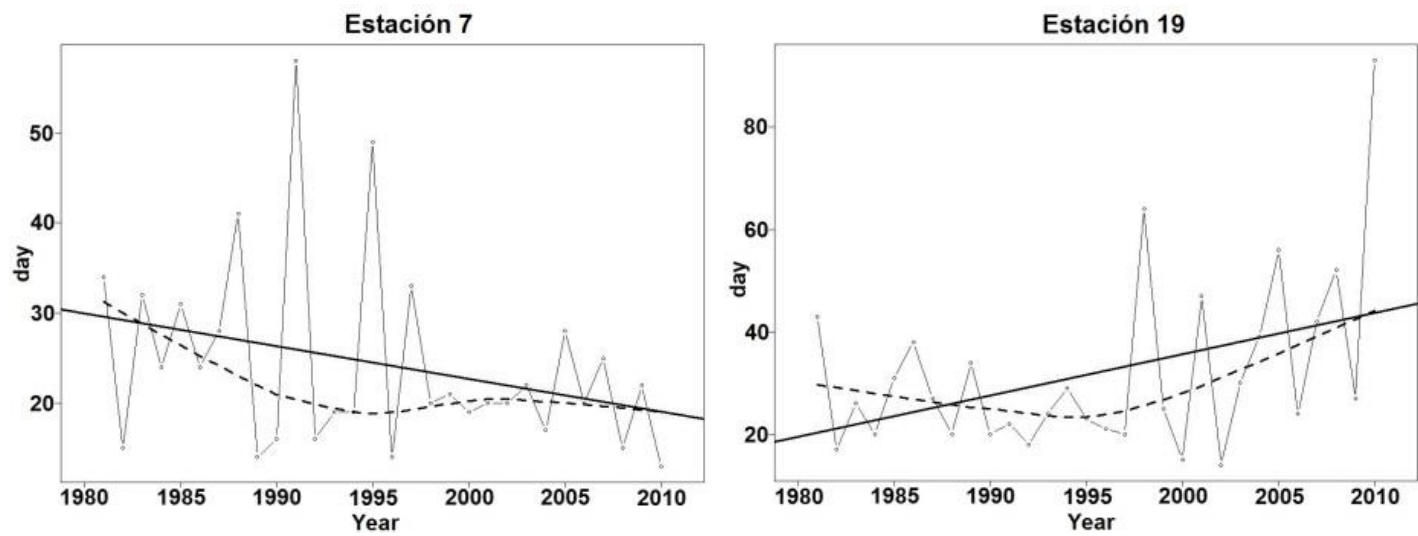

Fig. 12. Ejemplo de índice CDD.

Por otra parte los días húmedos consecutivos disminuyen en las estaciones 1 , $4,5,8,10,12,18$ y 21 con valores de hasta -0.73 días/década en la estación 4 (Figura 13) por el contrario para las 15 estaciones restantes aumenta hasta 1.56 días/década como en la estación 20 (Figura 13), lo que significa que los periodos secos serán mucho más prolongados, a pesar de que los periodos húmedos aumenten no lo harán al mismo ritmo que los periodos secos, reflejándose en que por cada década los días secos aumentarán hasta ocho días mientras que los días húmedos se incrementarán por mucho un día.
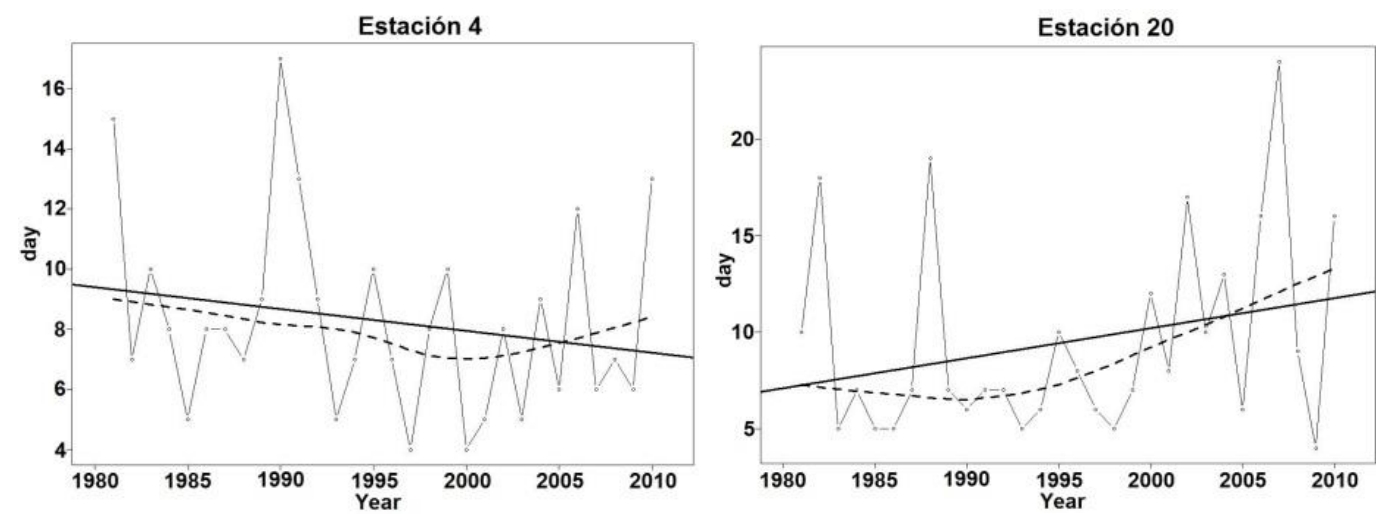

Fig. 13. Ejemplo de índice CWD.

El índice de días muy húmedos presenta una tasa de aumento entre 1.1 y 12.8 $\mathrm{mm} / \mathrm{año}$, para casi todas las estaciones, siendo el valor pico el de la estación 17 (Figura 14) con excepción de las estaciones 8 (Figura 14), 19, 20 que poseen tendencias de $-2.8,-0.4$ y $-1.7 \mathrm{~mm}$ /año respectivamente, estos valores se acercan a los propuestos por IDEAM [10]. Adicionalmente en la estación 8 a pesar de que la tendencia es negativa la oscilación de los valores de la última década es positiva. 

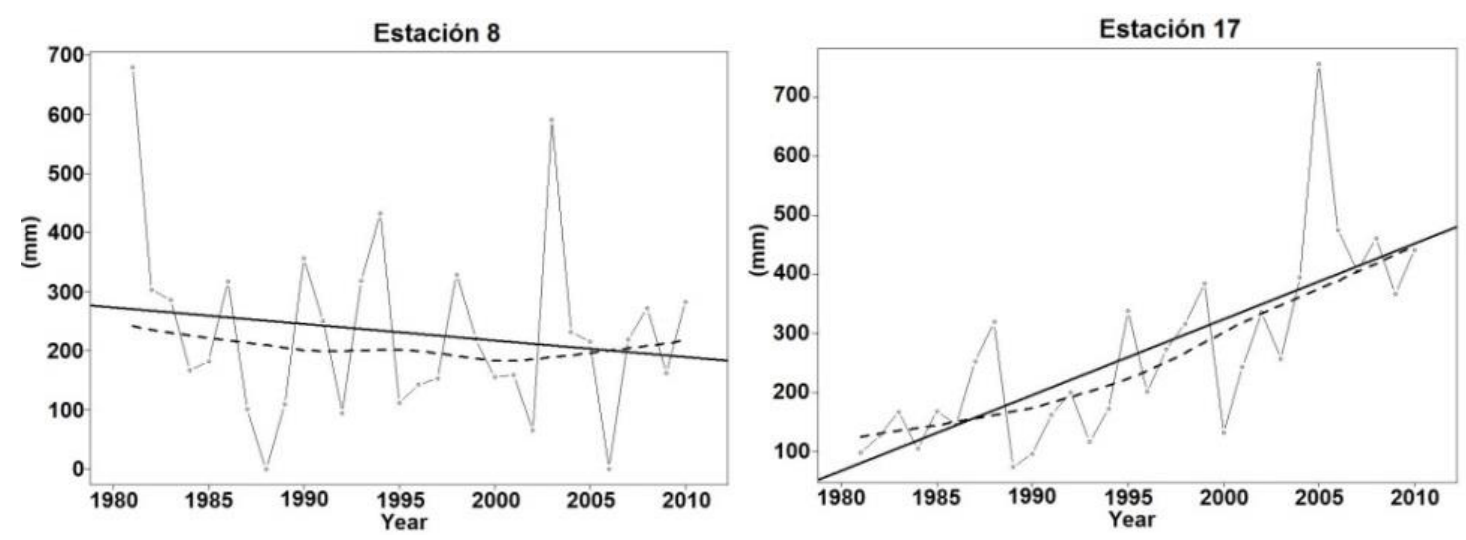

Fig. 14. Ejemplo de resultados índice R95p.

De manera similar los días extremadamente húmedos tienen tendencia positiva para casi todas las estaciones con valores de hasta $5.5 \mathrm{~mm} / \mathrm{año}$ como en la estación 17 (Figura 15) con excepción de las estaciones 8 (Figura 15) y estación 20 con tendencias de -1.7 y $-1.1 \mathrm{~mm} / \mathrm{año}$ respectivamente, lo que significa que los eventos que ocurren solo el $5 \%$ y el $1 \%$ de las veces en un año aumentaran en volumen de lluvia de una década a otra hasta $120 \mathrm{~mm}$ y 50 $\mathrm{mm}$ respectivamente, esperando que la magnitud de estos eventos sea más fuerte año tras año.
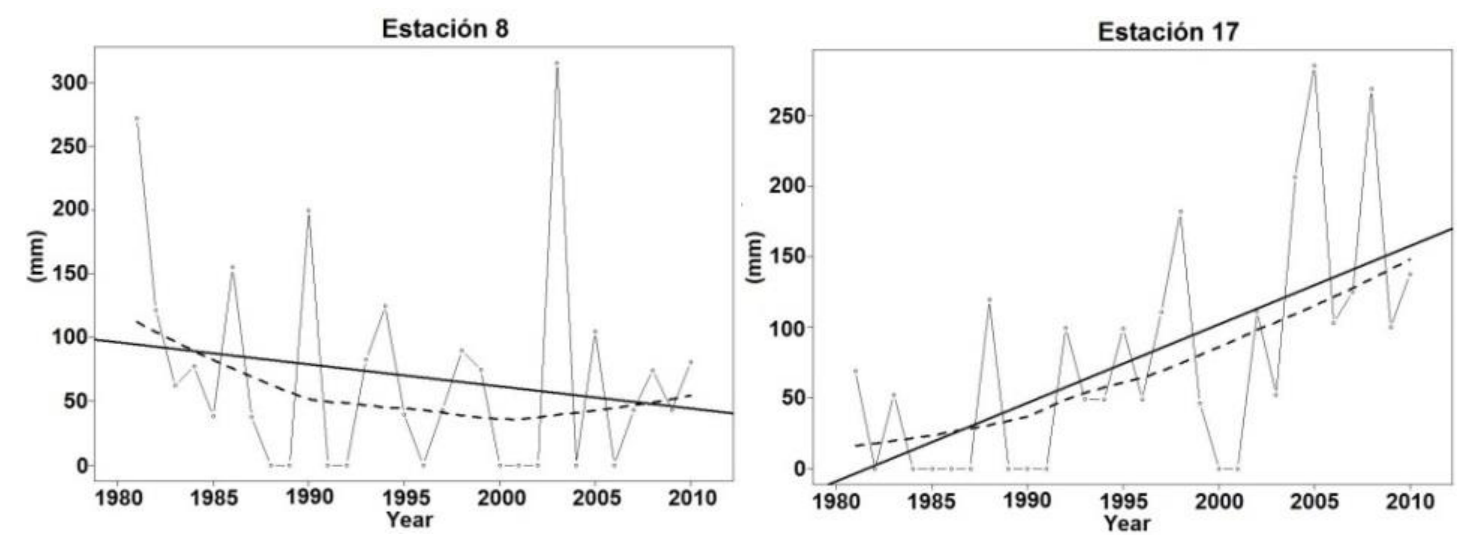

Fig. 15. Ejemplo de resultados índice R99.

El último índice revela la tendencia de precipitación total anual únicamente para los días húmedos que en su mayoría es positiva, presenta un crecimiento entre 4.5 y $15.8 \mathrm{~mm} / a n ̃ o$, siendo este último dato el valor pico a la estación 17 (Figura 16) con la excepción de las estaciones 8 (Figura 16), 19 y 20 que poseen tendencias de $-7.2,-0.4$ y $-1.1 \mathrm{~mm} /$ año respectivamente, es así como el volumen de lluvia aumentará en una proporción del doble cada año con respecto a la tendencia de descenso de precipitación, valores que incluso superan a los reportados por IDEAM [10], debido a que en dicha investigación el número de estaciones utilizadas fue menor. 
Análisis espacio temporal (1981-2010) de la precipitación en la ciudad de Bogotá: avances en la generación de índices extremos
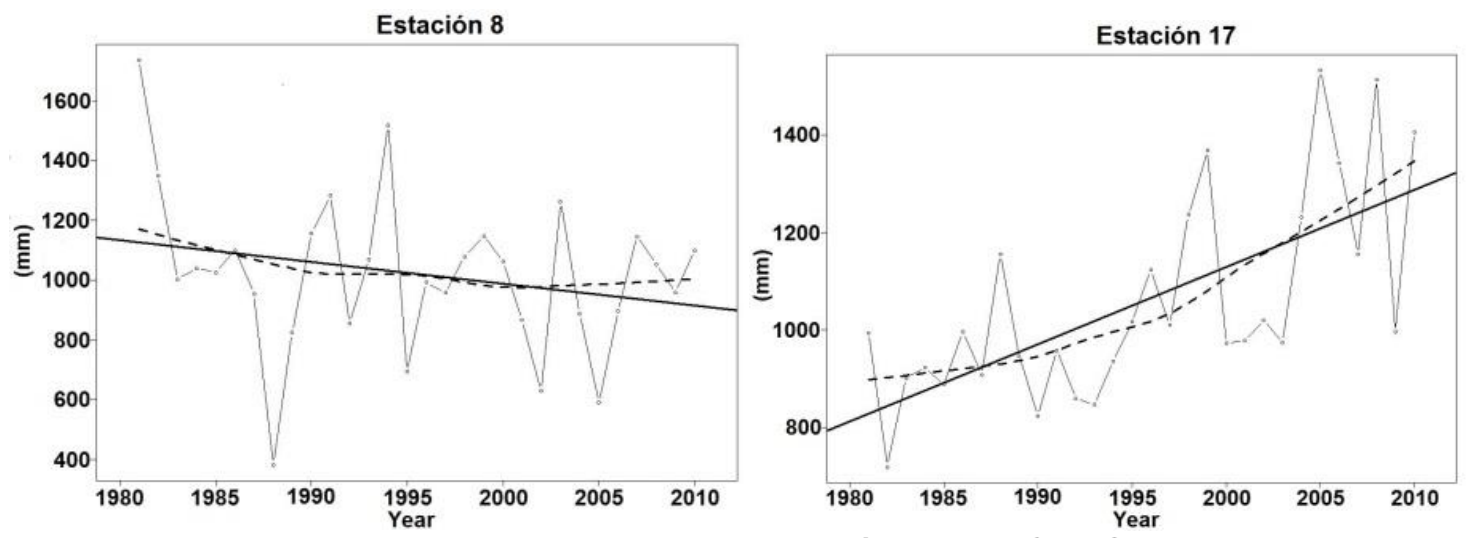

Fig. 16. Ejemplo de resultados índice PRCPTOT.

\section{Espacialización de datos climatológicos}

Las cuatro interpolaciones trabajadas, describieron la información de manera diferente de acuerdo con la Figura 17. Es así, como la interpolación Spline generó valores demasiado alejados de los datos de entrada y en el método de vecino natural el suavizado en las curvas fue muy bajo y adicional a ello el contorno de interpolación fue muy reducido. A su vez al evaluar el método Kriging lineal, el suavizado de las isoyetas no fue equitativo y extrapoló en exceso los datos, no coincidió con Di Piazza et al. [19], pero si coincidió con Vargas et al. [18] en que el método (IDW) no presentó los inconvenientes de los métodos anteriores por lo que fue escogido como el más adecuado para presentar la información climatológica.

En la zona oriental de la ciudad se presentó el mayor nivel de precipitación media anual, a medida que se desciende hacia zonas urbanas la precipitación empieza a disminuir, en el suroriente la precipitación no desciende tanto debido a la cadena montañosa. Por otra parte, la precipitación se extiende de manera aislada un poco hacia el centro de la ciudad donde se reduce la superficie de suelo asfaltada por parques y zonas boscosas como el Parque Simón Bolívar y el Jardín Botánico. Como se muestra en la Figura 17 los rangos de precipitación anual han presentado un aumento con respecto a lo reportado por IDEAM \& FOPAE [24], salvo la estación 20 que ha presentado un descenso dada la poca estabilidad y confiablidad de los datos de dicha estación. 

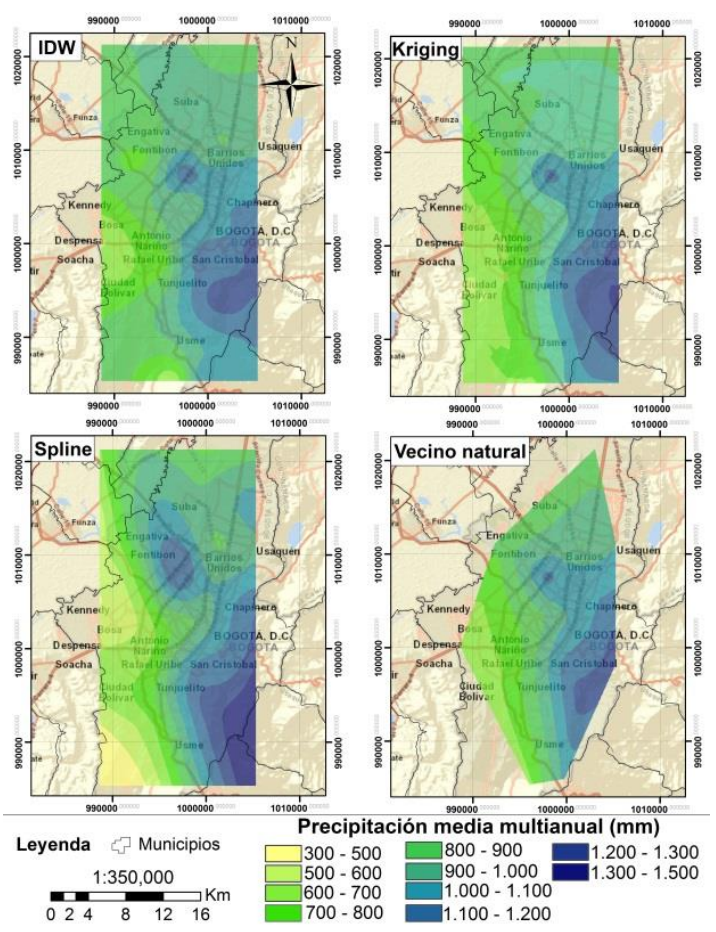

Fig. 17. Mapa de interpolaciones de precipitación media anual.
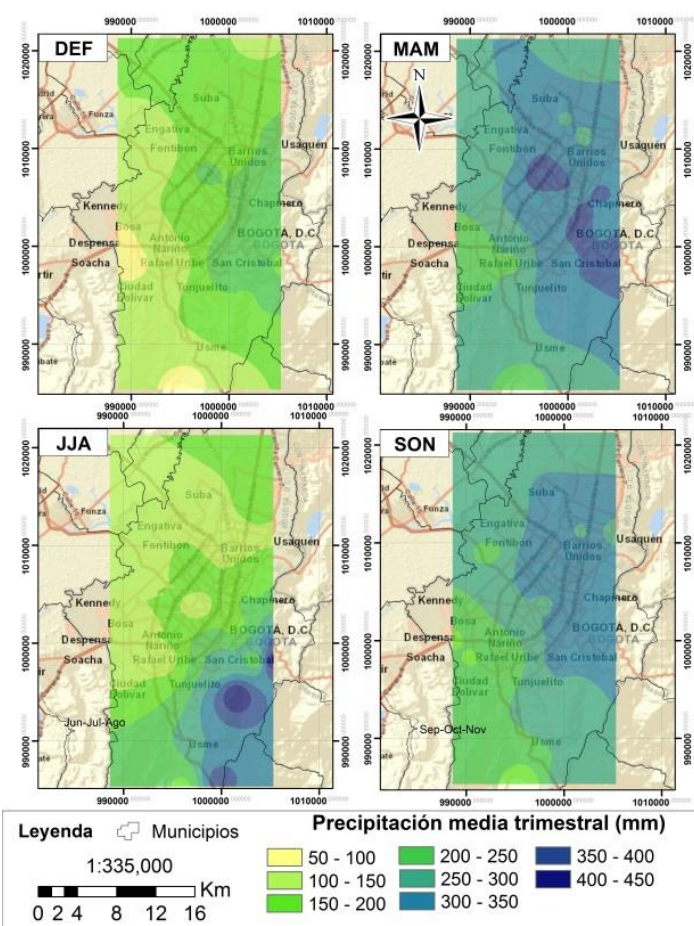

Fig. 18. Mapa de precipitación trimestral media.

En relación con lo anterior, la precipitación ha presentado cambios con respecto a la normal anterior, sin embargo, al utilizar la media anual hay información que se pierde y no permite analizar con un mayor detalle el comportamiento de la precipitación, para ello se obtuvo el mapa trimestral de precipitación media (Figura 18) que refleja lo dicho anteriormente en el análisis estadístico que los picos de precipitación trimestral son (JJA) para el grupo monomodal por tener más número días lluviosos, mientras que (MAM) y (SON) son los meses de mayor precipitación en el grupo bimodal.

Por otra parte, los índices de eventos extremos también se espacializaron, para de esta manera poder conocer el comportamiento geoespacial de cada uno. El primero corresponde a los índices Rx1day y Rx5day (Figura 19), reflejando que para un día de precipitación máxima el mayor volumen se encuentra en el oriente sin embargo se ve una leve tendencia de incremento hacia el occidente, esto último se evidencia mucho más cuando con cinco días consecutivos de lluvias intensas la precipitación se ha desplazado hacia el occidente dado que los cerros orientales forma una barrera natural que impiden seguir creciendo al oriente, ejemplo de ello es que en las estaciones 8 y 9 no presentaron valores tan altos dado que se ubican en la periferia oriental. 


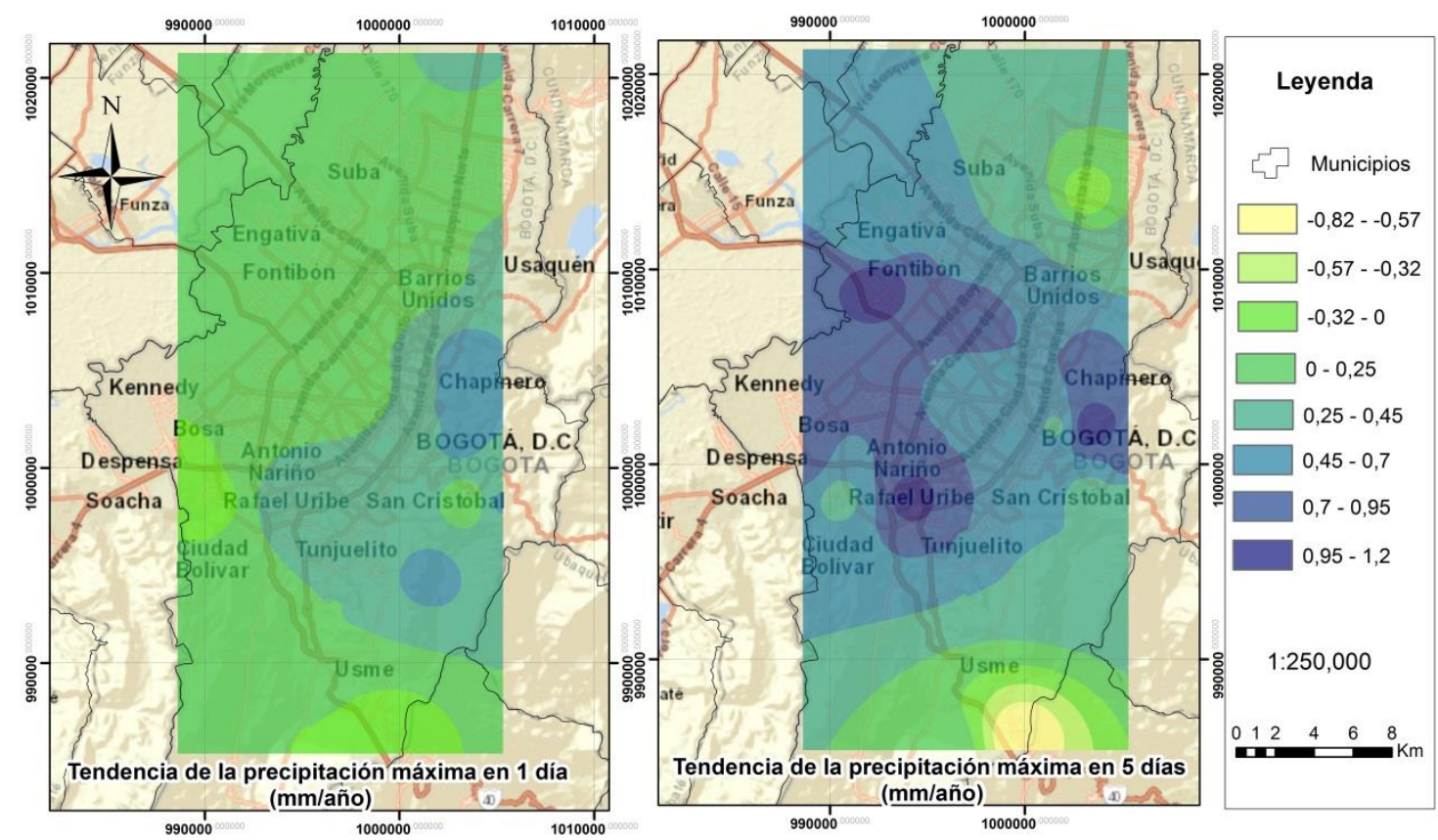

Fig. 19. Mapa de índices RX1day y RX5day.

En el mapa de índices R5, R10 y R20 (Figura 20) los eventos extremos se presentaron en el mismo sector para los tres índices, el cual corresponde a la parte urbana concéntrica y de comportamiento bimodal caso contrario en la zona monomodal la tendencia de aumentar los días con precipitaciones tan altas es muy baja, adicionalmente al sur se observan ondas de descenso, dicho de otra manera, se espera que en la zona urbana con mayor cantidad de edificios la tendencia de eventos extremos sea mayor que en zonas rurales.

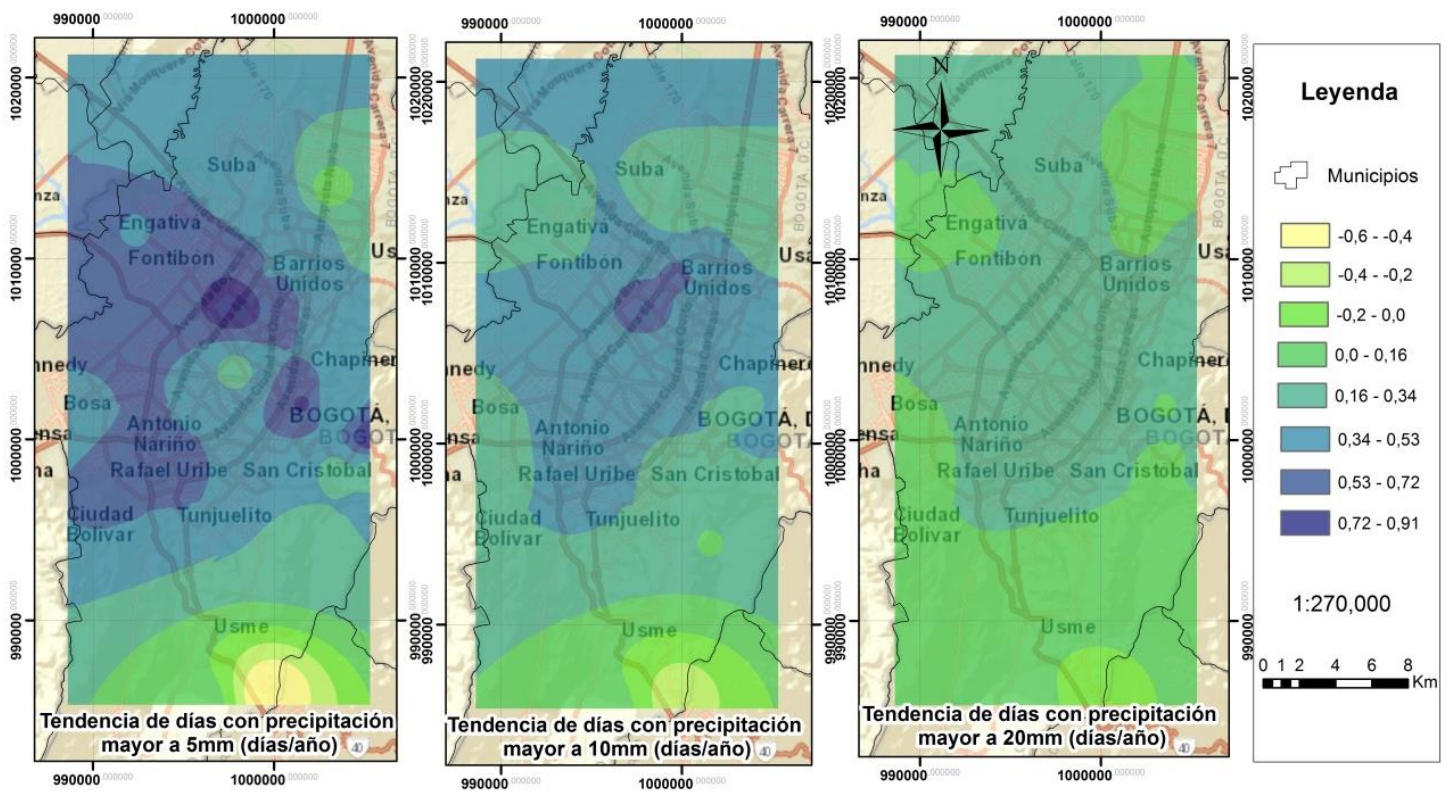

Fig. 20. Mapa de índices R5, R10 y R20.

En el mapa de días secos y húmedos consecutivos (Figura 21), en las zonas del sur la tendencia de prolongarse los periodos secos es mucho mayor que en el resto de la ciudad, de manera contraria en el centro, occidente y oriente la tendencia es negativa o muy cercana a cero y los periodos húmedos

Revista Facultad de Ingeniería (Rev. Fac. Ing.) Vol. 28 (51), pp. 51-71. Abril-Junio 2019. Tunja-Boyacá, Colombia. L-ISSN: 0121-1129, e-ISSN: 2357-5328, DOI: 
consecutivos para estas últimas zonas poseen una tendencia de aumento baja - cercana a cero, afirmando el hecho de que serán más prolongados los periodos secos, los eventos húmedos serán de mayor magnitud tal como se ha visto en los mapas anteriores.

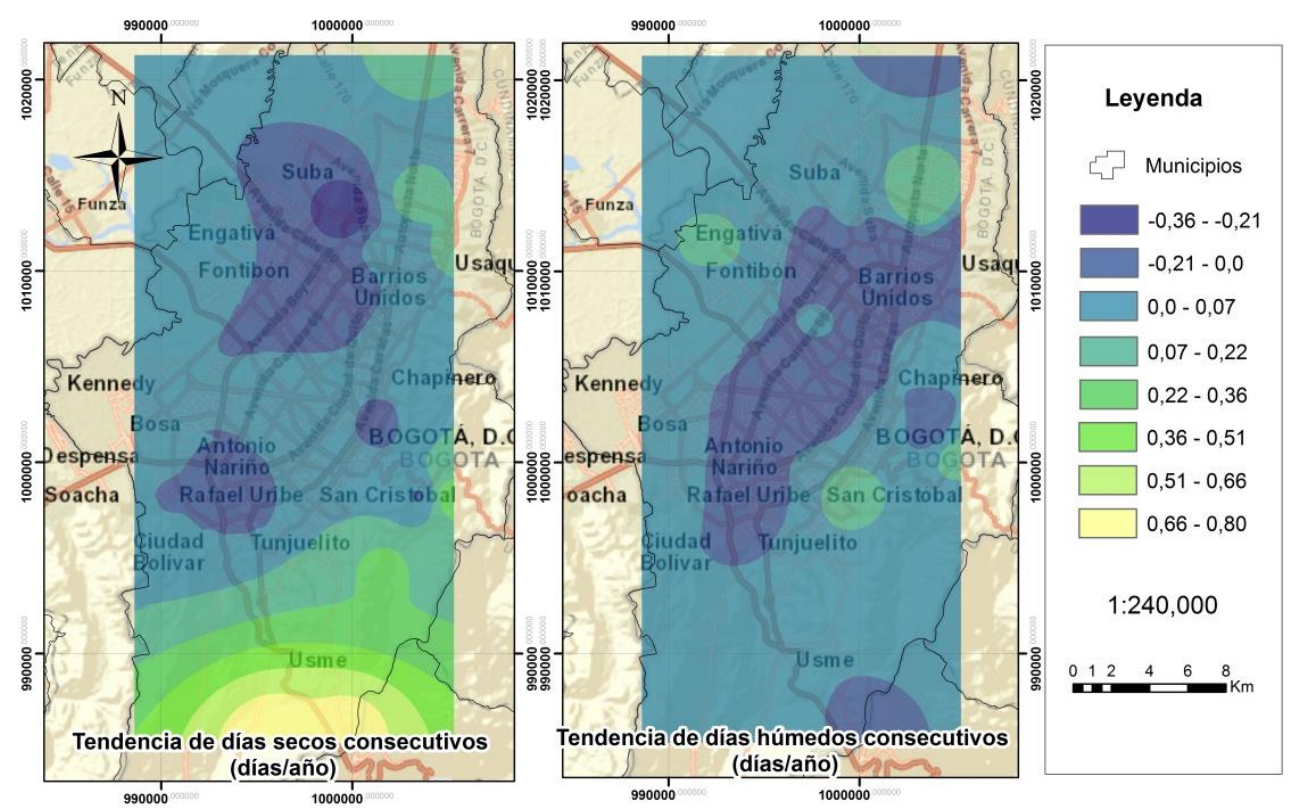

Fig. 21. Mapa de índices CDD y CWD.

La Figura 22 compara la información del índice con precipitaciones por encima del percentil 95 y 99, es decir eventos de baja ocurrencia, sin embargo, se caracterizan por un alto volumen de lluvia concentrado principalmente en el oriente, pero en la parte bimodal, presentando los máximos en las estaciones 10, 15 y 17, de esta manera se espera que los días muy húmedos aumenten en magnitud más que las lluvias extremas en gran parte la ciudad con excepción del sur y norte. Afirmando nuevamente que es más acelerado el crecimiento en volumen de lluvias en zonas con una fuerte presencia de edificios y superficie asfaltada que en zonas rurales con mayor cantidad de áreas boscosas. 
Análisis espacio temporal (1981-2010) de la precipitación en la ciudad de Bogotá: avances en la generación de índices extremos

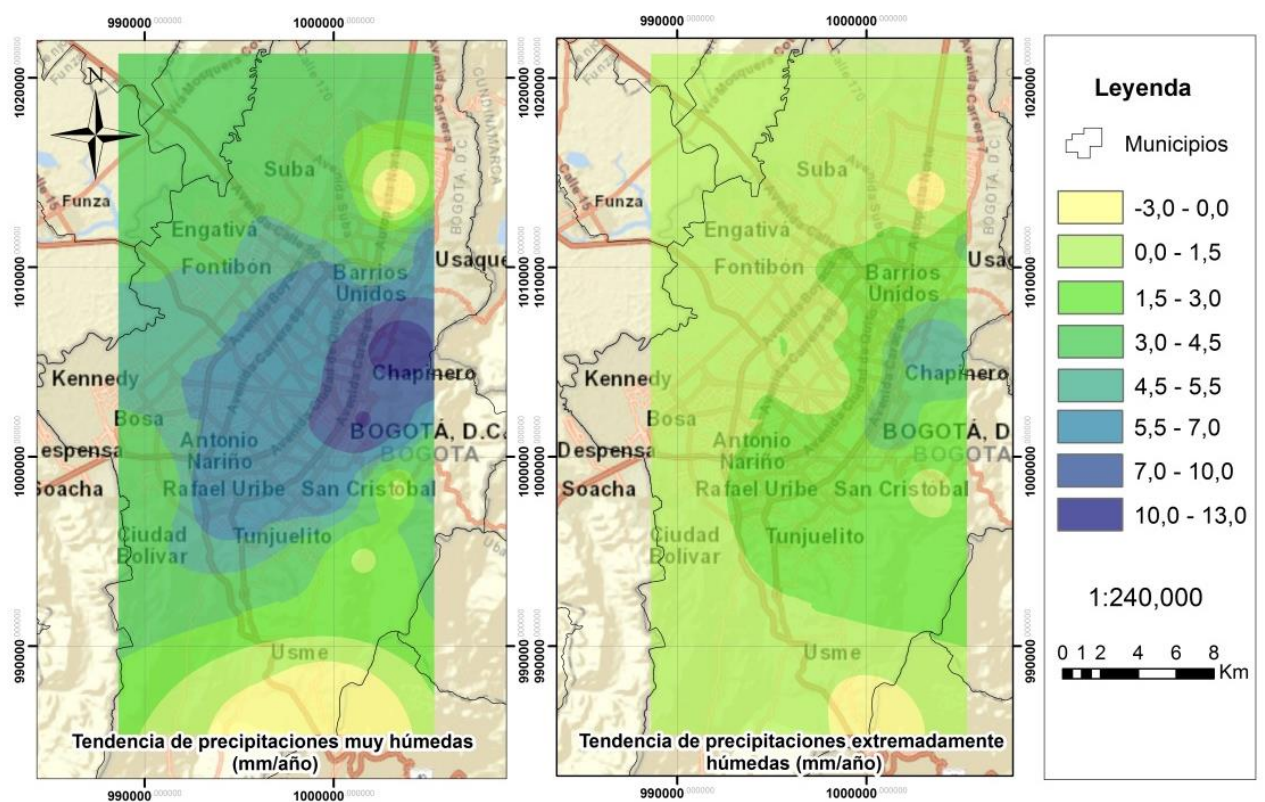

Fig. 22. Mapa de índices R95p y R99p.

El mapa de índice de tendencia de precipitación media en los días húmedos (Figura 23) revela que solo en la zona sur se presentará un descenso en el volumen de precipitación, coincidiendo con los mapas anteriores en que esta zona será la más seca de toda la ciudad, se observa además que la estación 20 posee un comportamiento bastante diferenciado con respecto a las estaciones cercanas, esto se atribuye a que tenía la mayor cantidad de datos faltantes, por lo que la confiabilidad de los datos no es alta. Por otra parte, la tendencia de aumento en volumen de lluvia se espera que en el resto de la ciudad sea más fuerte como por ejemplo en las estaciones 14, 15 y 17.
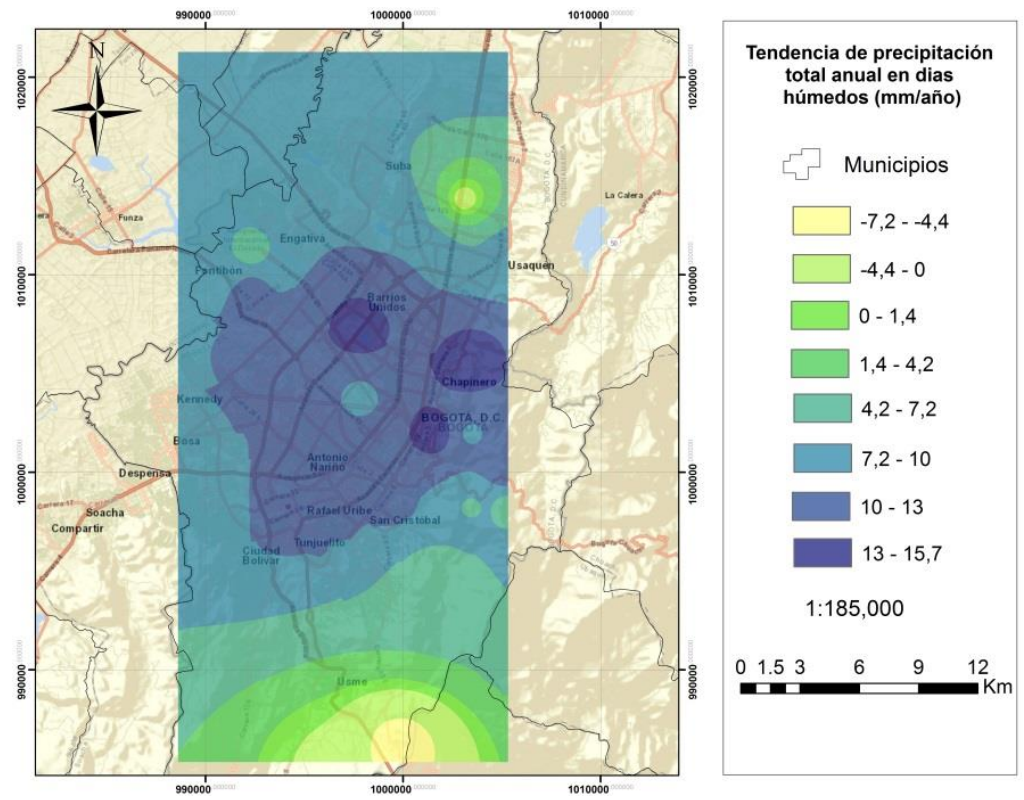

Fig. 23. Mapa de índice de precipitación total días húmedos.

\section{Conclusiones}

En Bogotá hacia la periferia rural suroriental, se esperan mayores lluvias en los meses de junio y julio, por lo que debe evaluarse el estado de alerta de estas

Revista Facultad de Ingeniería (Rev. Fac. Ing.) Vol. 28 (51), pp. 51-71. Abril-Junio 2019. Tunja-Boyacá, Colombia. L-ISSN: 0121-1129, e-ISSN: 2357-5328, DOI:

https://doi.org/10.19053/01211129.v28.n51.2019.9123 
zonas ante emergencias por deslizamientos dado que es una zona de alta pendiente. Es conveniente que, en la restante área de estudio, en los dos periodos lluviosos se tenga en cuenta el volumen de precipitación para la zonificación urbana y la prevención de inundaciones durante los diseños hidráulicos

En el área de estudio el primer indicador analizado, $\mathrm{RX} 1$, revela una mayor prioridad de análisis, para quienes toman decisiones, por representar un volumen mayor de precipitación en menor cantidad de tiempo. Lo anterior se complementa, con que habrá más distancia entre días húmedos separados por varios días secos. Con excepción del sur de la ciudad donde la tendencia es que la precipitación disminuya, los periodos secos serán más amplios presentando eventos extremos de sequía. Para la periferia rural sur, ubicada sobre los cerros orientales, la tendencia de aumentar tanto los días secos como húmedos es menor a un día por década, por lo que no se esperan eventos extremos.

Por último, la interpolación IDW es la que mejor se ajusta a la precipitación para Bogotá, sin generar sobreestimaciones de datos alejados de los reales, además se pudieron identificar dos zonas de gran convergencia de lluvias o islas de lluvias, la zona oriental y la zona central concéntrica a la estación Jardín Botánico, influenciadas principalmente por el área de cobertura vegetal que las rodea, recalcando la importancia de preservar y expandir estas zonas reguladoras climáticas.

\section{AGRADECIMIENTOS}

Los autores agradecen al Ingeniero Fabio Rubiano de la EAAB por la información de las estaciones meteorológica, a Lizeth Llanos investigadora del Centro Internacional de Agricultura Tropical (CIAT) por su asesoría en el uso del RClimtool, de igual manera al IDEAM y la CAR por suministrar la información meteorológica pertinente.

\section{REFERENCIAS}

[1] T. Rosmann, E. Dominguez, and J. Chavarro, "Comparing trends in hydrometeorological average andextreme data sets around the world at different time scales,"Journal of Hydrology: Regional Studies, vol. 5, pp. 200-212, Mar. 2016. DOI: https://doi.org/10.1016/i.ejrh.2015.12.061.

[2] J. Spinoni, P. Barbosa, A. De Jager, N. McCormick, G. Naumann, J. Vogt, D. Magni, D. Masante, and M. Mazzeschi, "A new global database of meteorological drought events from1951 to 2016," Journal of Hydrology: Regional Studies, vol. 22, Apr. 2019. pp100593. DOI: https://doi.org/10.1016/j.ejrh.2019.100593.

[3] K. Breinl, and G. Di Baldassarre, "Space-time disaggregation of precipitation and temperature across different climates and spatial scales," Journal of Hydrology: Regional Studies, vol. 21, pp. 126-146, Feb. 2019. DOI: https://doi.org/10.1016/j.ejrh.2018.12.002.

[4] C. López-Velandia, "Análisis de la distribución espacial y temporal de la precipitación en la cuenca del río Chicú, Sabana de Bogotá, Colombia," Perspectiva Geográfica, vol. 21 (1), pp. 63-90, Jun. 2016. DOI: https://doi.org/10.19053/01233769.4545.

[5] C. Torres, and J. D. Pabón, "Variabilidad intraestacional de la precipitación en Colombia y su relación con la oscilación de Madden-Julian," Revista de la Academia Colombiana de Ciencias Exactas, Físicas y Naturales, vol. 158 (41), pp. 79-93, 2017. DOI: https://doi.org/10.18257/raccefyn.380.

[6] B. Olivares, "Tropical conditions of seasonal rain in the dry-land agriculture of Carabobo, Venezuela," La Granja: Revista de Ciencias de la Vida, vol. 27 (1), pp. 89-102, 2018. 
Análisis espacio temporal (1981-2010) de la precipitación en la ciudad de Bogotá: avances en la generación de índices extremos

[7] J. Pabón, and G. Torres, "Impacto socioeconómico de los fenómenos El Niño y La Niña en la Sabana de Bogotá durante el siglo XX," Cuadernos de Geografía: Revista Colombiana de Geografía, vol.16, pp. 81-94, 2007. DOI: https://doi.org/10.15446/rcdg.v22n2.37015.

[8] E. Cepeda, Recopilación histórica y análisis climatológico de eventos de granizada ocurridos en Bogotá y su relación con el cambio climático global, Doctoral Thesis, Universidad Nacional de Colombia, Bogotá, 2010.

[9] D. Pabon, and T. Beisiegel, "Pronóstico de granizadas mediante modelos numéricos regionales en la zona andina: El caso de la tormenta del 3 de noviembre de 2007 sobre Bogotá D.C.," In: IX Congreso colombiano de meteorología, Bogotá, Colombia, 2011.

[10] Instituto de Hidrología, Meteorología y Estudios Ambientales - IDEAM, Características climatológicas de ciudades principales y municipios turísticos, 2015. Available: http://www.ideam.gov.co/documents/21021/21789/1Sitios+turisticos2.pdf/cd4106e9-d608-4c2991cc-16bee9151ddd.

[11] D. E. Cuartas, D. M. Caicedo, D. Ortega, F. Cardona, Y. Carvajal, and F. Méndez, "Tendencia espacial y temporal de eventos climáticos extremos en el valle geográfico del Río Cauca," Revista $\begin{array}{llllll}\text { U.D.C.A., } & \text { vol. } 20 & \text { (2), } 2017 . & \text { pp. }\end{array}$ https://doi.org/10.31910/rudca.v20.n2.2017.386.

[12] A. Esquivel, L. Llanos, D. Agudelo, S. Prager, K. Fernandes, A. Rojas, V. Jhon, and J. Ramirez, "Predictability of seasonal precipitation across major crop growing areas in Colombia," Climate Services, vol. 12, pp. 36-47, Dec. 2018. DOI: https://doi.org/10.1016/i.cliser.2018.09.001.

[13] Instituto de Hidrología, Meteorología y Estudios Ambientales - IDEAM, Incorporación de la gestión adaptativa de los riesgos hidroclimáticos en el ordenamiento territorial, Plan Regional Integral de Cambio Climático Región Capital, Bogotá, Colombia, 2012.

[14] H. O. Benavides, R. Mayorga, and G. Hurtado, "Análisis de índices de extremos climáticos para Colombia usando el Rclimdex," Nota técnica IDEAM - METEO /007, 2007.

[15] Instituto de Hidrología, Meteorología y Estudios Ambientales - IDEAM, Señales de cambio climático por análisis de extremos climáticos, Plan Regional Integral de Cambio Climático de Bogotá, Bogotá, 2012.

[16] J. Losada, Análisis de posibles cambios a través del tiempo en la ocurrencia, intensidad y magnitud de la precipitación para tormentas extremas sobre la ciudad de Bogotá, Doctoral Thesis, Universidad Nacional de Colombia, Bogotá, 2015.

[17] A. Klein Tank, F. Zwiers, and X. Zhang, "Guidelines on Analysis of extremes in a changing climate in support of informed decisions for adaptation," WCDMP-72,WMO-TD/No. 1500, Ginebra, 2009.

[18] A. Vargas, A. Santos, E. Cárdenas, and N. Obregón, "Análisis de la distribución e interpolación espacial de las lluvias en Bogotá, Colombia," Dyna, vol. 78, pp. 150-158., 2011.

[19] A. Di Piazza, F. L. Conti, L. V. Noto, F. Viola, and G. La Loggia, "Análisis comparativo de diferentes técnicas para la interpolación espacial de datos de lluvia para crear una serie de series de precipitación mensuales completas en serie para Sicilia, Italia," Revista Internacional de Observación de la Tierra Aplicada, vol. 13 (3), pp. 396-408, 2011.

[20] M. Hutchinson, D. McKenney, K. Lawrence, J. Pedlar, R. Hopkinson, E. Milewska, and P. Papadopol, "Desarrollo y prueba de modelos espaciales interpolados de temperatura y precipitación diaria mínima-máxima de Canadá para 1961-2003," Diario de Meteorología Aplicada y Climatología, vol. 48 (4), pp. 725 - 741, 2009. DOI: https://doi.org/10.1175/2008jamc1979.1.

[21] Instituto Geográfico Agustín Codazzi - IGAC, "Tipos de coordenadas manejadas en Colombia," 2014.

Available: http://www2.igac.gov.co/igac web/UserFiles/File/MAGNAWEB final/documentos/tipos\%20de\%20c oordenadas.pdf.

[22] J. Cárdenas, Análisis geoestadistico del comportamiento de la temperatura en la ciudad de Bogotá desde el año 1981 al 2010, Grade Thesis, Universidad Libre, Bogotá, 2016.

[23] V. L. López, "Condiciones atmosféricas predominantes en el piedemonte de la cordillera Oriental, determinantes del régimen climático en las sedes de la Universidad de La Salle, Bogotá," Épsilon, vol. 18, pp. 87-104, 2012.

[24] G. Bernal, M. Rosero, M. Cadena, J. Montealegre, and F. Sanabria, Estudio de la Caracterización Climática de Bogotá y Cuenca Alta del Río Tunjuelo, Instituto de Hidrología, Meteorología y Estudios Ambientales IDEAM - Fondo de Prevención y Atención de Emergencias - FOPAE, Bogotá, 2007.

[25] J. D. Pabón, and G. A. Torres, "Efecto climático de los fenómenos el Niño y la Niña en la sabana de Bogotá," Meteorología Colombiana, vol. 18, pp. 86-99, 2006.

[26] J. Ramirez-Villegas, and A. Challinor, "Assessing relevant climate data for agricultural applications," Agricultural and Forest Meteorology, no 161, pp. 26-45, 2012 . DOI: https://doi.org/10.1016/j.agrformet.2012.03.015.

Revista Facultad de Ingeniería (Rev. Fac. Ing.) Vol. 28 (51), pp. 51-71. Abril-Junio 2019. Tunja-Boyacá, Colombia. L-ISSN: 0121-1129, e-ISSN: 2357-5328, DOI: https://doi.org/10.19053/01211129.v28.n51.2019.9123 
[27] B. Olivares, A. Cortez, R. Parra, M. Rodriguez, and E. Guevara, "Aplicación de procedimientos estadísticos para el control de calidad de las series de precipitación mensual en los llanos orientales venezolanos," Revista Facultad de Agronomia, vol. 30, pp. 367-391, 2013.

[28] L. Llanos, "RClimtool," 2014. Available: http://www.aclimatecolombia.org/rclimtool-free-applicationanalyzing-climatic-series/.

[29] Instituto de Hidrología, Meteorología y Estudios Ambientales - IDEAM, "Atlas climatológico, radiación y viento," 2018. Available: http://atlas.ideam.gov.co/visorAtlasClimatologico.html.

[30] OMM, Guía de prácticas climatológicas, Ginebra: Organización Meteorológica Mundial, 2011.

[31] A. Barrera, Técnicas de completado de series mensuales y aplicación al estudio de la influencia de la NAO en la distribución de la precipitación en España, Doctoral Thesis, Barcelona, Universidad de Barcelona, 2004.

[32] T. C. Peterson, and D. R. Easterling, "Creation of homogeneous composite climatological reference series," International Journal of Climatology, vol. 14 (6), pp. 671-679, 1994. DOI: https://doi.org/10.1002/joc.3370140606.

[33] J. E. Torres, and J. A. Aragón, Investigación en hidrología: general, isotópica y aplicada, Grade Thesis, Bogotá: Universidad Libre, 2018.

[34] NOAA, "Precipitation Measurements," 2017. Available: https://www.weather.gov/abrfc/map.

[35] D. Wilks, Statistical Methods in the Atmopheric Sciences, United States of America: Elsevier, 2006.

[36] J. W. Tukey, Exploratory Data Analysis, 1977.

[37] J. L. Santos, RClimdex (1.0), Centro internacional para la investigación del fenómeno de El Niño (CIIFEN), 2004.

[38] OMM, "Report on the Activities of the Working Group on Climate Change Detection and Related Rapporteurs 1998-2001," WMO/TD-No. 1071/WCDMP-No. 47, Ginebra, 2001. 